\title{
TRIGONOMETRIC DEGENERATION AND ORBIFOLD WESS-ZUMINO-WITTEN MODEL. II
}

\author{
TAKASHI TAKEBE \\ Dedicated to Professor Akihiro Tsuchiya on his 60th birthday.
}

\begin{abstract}
The sheaves of conformal blocks and conformal coinvariants of the twisted WZW model have a factorisation property and are locally free even at the boundary of the moduli space, where the elliptic KZ equations and the Baxter-Belavin elliptic $r$ matrix degenerate to the trigonometric $\mathrm{KZ}$ equations and the trigonometric $r$ matrix, respectively. Etingof's construction of the elliptic KZ equations is geometrically interpreted.
\end{abstract}

\section{INTRODUCTION}

This is a continuation of the paper $[\mathbf{T}$. We showed there that the trigonometric WZW model is factorised into the orbifold WZW models. Using this result, we show in the present article that the trigonometric WZW model is indeed the degenerate twisted WZW models on elliptic curves defined in [KT]. More precisely, we prove that there are locally free sheaves over the partially compactified family of elliptic curves, the fibre of which are the space of conformal blocks or the space of conformal coinvariants of the twisted WZW model at a generic point and those of the trigonometric WZW model at the discriminant locus, when all inserted modules are either Weyl modules (Proposition 4.2) or integrable highest weight modules (Theorem 6.1).

Since the elliptic $r$ matrix (cf. $[\mathrm{BD}$, E]) describing the elliptic KZ equations degenerates to the trigonometric $r$ matrix, the above fact is naturally expected, though rigorous proof requires careful algebrogeometric arguments as in TUY].

2000 Mathematics Subject Classification. Primary 81T40; Secondary 14H15, 17B67, 17B81, 32G15.

Key words and phrases. Trigonometric degeneration; twisted WZW model; orbifold WZW model; factorisation.

This work is partly supported by the Grant-in-Aid for Scientific Research (C) of the Japan Society for the Promotion of Science, No. 15540014. 
The paper is organised as follows. After reviewing the twisted WZW model on elliptic curves in $\$ 2$ to recall basic notions and notations, we define family of elliptic curves with a singular fibre and a twisted Lie algebra bundle over it in $\$ 3$ In $\$ 4$ main objects of this paper, the sheaves of conformal coinvariants and conformal blocks, are defined and their coherence is proved. In particular when all the modules inserted to the curve are Weyl modules, they are locally free. To prove the locally freeness of the sheaf of conformal coinvariants for integrable highest weight modules, we examine its behaviour at the discriminant locus. In this case the factorisation theorem, Theorem 7 of [T], is refined in $\$ 5$. The proof of locally freeness in $\$ 6$ follows the strategy of [TUY] and [TK].

Notations. We use the following notations besides other ordinary conventions in mathematics.

- $N$, L: fixed integers. $N \geqq 2$ will be the matrix size and $L \geqq 1$ will be the number of the marked points on a curve.

- $C_{N}:=\mathbb{Z} / N \mathbb{Z}$ : the cyclic group of order $N$.

- When $X$ is an algebraic variety, $\mathcal{O}_{X}$ denotes the structure sheaf of $X$. When $P$ is a point on $X$ and $\mathcal{F}$ is an $\mathcal{O}_{X}$-sheaf, $\mathcal{F}_{P}$ denotes the stalk of $\mathcal{F}$ at $P . \mathfrak{m}_{P}$ is the maximal ideal of the local $\operatorname{ring} \mathcal{O}_{X, P} .\left.\quad \mathcal{F}\right|_{P}:=\mathcal{F}_{P} / \mathfrak{m}_{P} \mathcal{F}_{P}, \mathcal{F}_{P}^{\wedge}=\operatorname{proj} \lim _{n \rightarrow \infty} \mathcal{F}_{P} / \mathfrak{m}_{P}^{n} \mathcal{F}_{P}$ are the fibre of $\mathcal{F}$ at $P$ and the $\mathfrak{m}_{P}$-adic completion of $\mathcal{F}_{P}$, respectively.

- We shall use the same symbol for a vector bundle and for a locally free $\mathcal{O}_{X}$-module consisting of its local holomorphic sections.

\section{TWisted WZW MODEL ON ELLIPTIC CURVES}

In this section we briefly review the twisted WZW model on elliptic curves. See [KT for details.

We fix an invariant inner product of $\mathfrak{g}=s l_{N}(\mathbb{C})$ by

$$
(A \mid B):=\operatorname{tr}(A B) \quad \text { for } A, B \in \mathfrak{g} .
$$

Define matrices $\beta$ and $\gamma$ by

(2) $\beta:=\left(\begin{array}{cccc}0 & 1 & & 0 \\ & 0 & \ddots & \\ & & \ddots & 1 \\ 1 & & & 0\end{array}\right), \quad \gamma:=\left(\begin{array}{cccc}1 & & & 0 \\ & \varepsilon^{-1} & & \\ & & \ddots & \\ 0 & & & \varepsilon^{1-N}\end{array}\right)$,

where $\varepsilon=\exp (2 \pi i / N)$. Then we have $\beta^{N}=\gamma^{N}=1$ and $\gamma \beta=\varepsilon \beta \gamma$. 
Let $E=E_{\tau}$ be the elliptic curve with modulus $\tau: E_{\tau}:=\mathbb{C} / \mathbb{Z}+\tau \mathbb{Z}$. We define a Lie algebra bundle $\mathfrak{g}^{\text {tw }}$ with fibre $\mathfrak{g}=s l_{N}(\mathbb{C})$ over $E$ by

$$
\mathfrak{g}^{\mathrm{tw}}:=(\mathbb{C} \times \mathfrak{g}) / \approx
$$

where the equivalence relations $\approx$ are defined by

$$
(z, A) \approx(z+1, \operatorname{Ad} \gamma(A)) \approx(z+\tau, \operatorname{Ad} \beta(A)) .
$$

Let $J_{a b}=\beta^{a} \gamma^{-b}$, which satisfies

$$
\operatorname{Ad} \gamma\left(J_{a b}\right)=\varepsilon^{a} J_{a b}, \quad \operatorname{Ad} \beta\left(J_{a b}\right)=\varepsilon^{b} J_{a b} .
$$

Global meromorphic sections of $\mathfrak{g}^{\mathrm{tw}}$ are linear combinations of $J_{a b} f(z)$ $(a, b=0, \ldots, N-1,(a, b) \neq(0,0))$, where $f(z)$ is a meromorphic function with quasi-periodicity,

$$
f(z+1)=\varepsilon^{a} f(z), \quad f(z+\tau)=\varepsilon^{b} f(z) .
$$

For each point $P$ on $E$, we define a Lie algebra,

$$
\mathfrak{g}^{P}:=\left(\mathfrak{g}^{\mathrm{tw}} \otimes_{\mathcal{O}_{E}} \mathcal{K}_{E}\right)_{P}^{\wedge}
$$

where $\mathcal{K}_{E}$ is the sheaf of meromorphic functions on $E$ and $(\cdot)_{P}^{\wedge}$ means the completion of the stalk at $P$ with respect to the maximal ideal $\mathfrak{m}_{P}$ of $\mathcal{O}_{E, P}$, the stalk of the structure sheaf. The Lie algebra $\mathfrak{g}^{P}$ is (noncanonically) isomorphic to the loop Lie algebra $\mathfrak{g}\left(\left(z-z_{0}\right)\right)$, where $z_{0}$ is the coordinate of $P$. The subspace

$$
\mathfrak{g}_{+}^{P}:=\left(\mathfrak{g}^{\mathrm{tw}}\right)_{P}^{\wedge} \cong \mathfrak{g}\left[\left[z-z_{0}\right]\right]
$$

of $\mathfrak{g}^{P}$ is a Lie subalgebra.

Let us fix mutually distinct points $Q_{1}, \ldots, Q_{L}$ on $E$ whose coordinates are $z=z_{1}, \ldots, z_{L}$ and put $D:=\left\{Q_{1}, \ldots, Q_{L}\right\}$. We shall also regard $D$ as a divisor on $E$ (i.e., $D=Q_{1}+\cdots+Q_{L}$ ). The Lie algebra $\mathfrak{g}^{D}:=\bigoplus_{i=1}^{L} \mathfrak{g}^{Q_{i}}$ has a 2-cocycle defined by

$$
\mathrm{c}_{\mathrm{a}}(A, B):=\sum_{i=1}^{L} \mathrm{c}_{\mathrm{a}, i}\left(A_{i}, B_{i}\right), \quad \mathrm{c}_{\mathrm{a}, i}\left(A_{i}, B_{i}\right):=\operatorname{Res}_{Q_{i}}\left(d A_{i} \mid B_{i}\right),
$$

where $A=\left(A_{i}\right)_{i=1}^{L}, B=\left(B_{i}\right)_{i=1}^{L} \in \mathfrak{g}^{D}, \operatorname{Res}_{Q_{i}}$ is the residue at $Q_{i}$ and $d$ is the exterior derivation. (The symbol "c ${ }_{\mathrm{a}}$ " stands for "Cocycle defining the Affine Lie algebra".) We denote the central extension of $\mathfrak{g}^{D}$ with respect to this cocycle by $\hat{\mathfrak{g}}^{D}$ :

$$
\hat{\mathfrak{g}}^{D}:=\mathfrak{g}^{D} \oplus \mathbb{C} \hat{k}
$$

where $\hat{k}$ is a central element. Explicitly the bracket of $\hat{\mathfrak{g}}^{D}$ is represented as

$$
[A, B]=\left(\left[A_{i}, B_{i}\right]^{\circ}\right)_{i=1}^{L} \oplus \mathrm{c}_{\mathrm{a}}(A, B) \hat{k} \quad \text { for } A, B \in \mathfrak{g}^{D}
$$


where $\left[A_{i}, B_{i}\right]^{\circ}$ are the natural bracket in $\mathfrak{g}^{Q_{i}}$. The Lie algebra $\hat{\mathfrak{g}}^{P}$ for a point $P$ is nothing but the affine Lie algebra $\hat{\mathfrak{g}}$ of type $A_{N-1}^{(1)}$ (a central extension of the loop algebra $\left.\mathfrak{g}((t-z))=s l_{N}(\mathbb{C}((t-z)))\right)$.

The affine Lie algebra $\hat{\mathfrak{g}}^{Q_{i}}$ can be regarded as a subalgebra of $\hat{\mathfrak{g}}^{D}$. The subalgebra $\mathfrak{g}_{+}^{Q_{i}}$ of $\mathfrak{g}^{Q_{i}}$ (cf. (8) ) can be also regarded as a subalgebra of $\hat{\mathfrak{g}}^{Q_{i}}$ and $\hat{\mathfrak{g}}^{D}$.

Let $\mathfrak{g}_{\text {out }}$ be the space of global meromorphic sections of $\mathfrak{g}^{\text {tw }}$ which are holomorphic on $E$ except at $D$ :

$$
\mathfrak{g}_{\text {out }}:=\Gamma\left(E, \mathfrak{g}^{\mathrm{tw}}(* D)\right) .
$$

The residue theorem implies that we can regard $\mathfrak{g}_{\text {out }}$ as a Lie subalgebra of $\hat{\mathfrak{g}}^{D}$ by mapping an element of $\mathfrak{g}_{\text {out }}$ to its germs at $Q_{i}$ 's.

Definition 2.1. The space of conformal coinvariants $\mathrm{CC}_{E}(M)$ and that of conformal blocks $\mathrm{CB}_{E}(M)$ over $E$ associated to $\hat{\mathfrak{g}}^{Q_{i}}$-modules $M_{i}$ with the same level $\hat{k}=k$ are defined by

$$
\mathrm{CC}_{E}(M):=M / \mathfrak{g}_{\text {out }} M, \quad \mathrm{CB}_{E}(M):=\operatorname{Hom}_{\mathbb{C}}\left(M / \mathfrak{g}_{\text {out }} M, \mathbb{C}\right),
$$

where $M:=\bigotimes_{i=1}^{L} M_{i}$. The module $M_{i}$ is referred to as "a module inserted at the point $Q_{i} "$.

\section{FAMily OF ELLIPTIC CURVES}

In this section we construct a family of elliptic curves $\mathcal{E}$ with a singular fibre and its covering $\tilde{\mathcal{E}}$. A twisted Lie algebra bundle $\mathfrak{g}^{\text {tw }}$ over the generic fibres of $\mathcal{E}$ is one of the main representation theoretical data in the twisted WZW model but it does not directly extends to the singular fibre. Hence we pull it back to $\tilde{\mathcal{E}}$ and trivialise it. The sections of $\mathfrak{g}^{\mathrm{tw}}$ on the singular fibre is understood as sections of the trivial bundle on $\tilde{\mathcal{E}}$ invariant under the action of the covering transformation group.

The construction of $\tilde{\mathcal{E}}$ is almost the same as that of the analytic fibre space of elliptic curves in $\left[\mathrm{Wo}\right.$. We use $N$ patches $U_{k}(k \in \mathbb{Z} / N \mathbb{Z})$ :

$$
U_{k}:=\left\{\left.\left(q, x_{k}, y_{k}\right)|| q\left|<1, x_{k} y_{k}=q,\right| x_{k}|<| q\right|^{-1},\left|y_{k}\right|<|q|^{-1}\right\} .
$$

We denote $(q, x, y) \in U_{k}$ by $(q, x, y)_{k}$. The universal curve $\tilde{\mathcal{E}}$ is defined by

$$
\tilde{\mathcal{E}}:=\bigsqcup_{k \in \mathbb{Z} / N \mathbb{Z}} U_{k} / \sim,
$$

where the equivalence relation $\sim$ is defined by

$$
\left(q, x_{k}, y_{k}\right)_{k} \sim\left(q, x_{k+1}^{\prime}, y_{k+1}^{\prime}\right)_{k+1} \text { when } x_{k} y_{k+1}^{\prime}=1 \text {. }
$$


We have an analytic fibre space $\tilde{\pi}: \tilde{\mathcal{E}} \rightarrow \Delta$ over $\Delta=\{q|| q \mid<1\}$. The fibre over $q \neq 0$ is an elliptic curve $\mathbb{C}^{\times} / q^{N \mathbb{Z}}$ and the fibre over $q=0$ is singular with ordinary double points $(0,0,0)_{k} \in U_{k}(k \in \mathbb{Z} / N \mathbb{Z})$.

Let $C_{N}=\{\overline{0}, \overline{1}, \ldots, \overline{N-1}\}$ be the cyclic group of order $N$. The group $C_{N}^{2}=C_{N} \times C_{N}$ acts on $\tilde{\mathcal{E}}$ from the right as follows: it is enough to define the action of the generators $(1,0)$ and $(0,1)$ of $C_{N}^{2}$.

$\left(q, x_{k}, y_{k}\right)_{k} \cdot(1,0)=\left(q, \varepsilon^{-1} x_{k}, \varepsilon y_{k}\right)_{k}, \quad\left(q, x_{k}, y_{k}\right)_{k} \cdot(0,1)=\left(q, x_{k}, y_{k}\right)_{k-1}$.

The universal curve $\mathcal{E}$ is defined set-theoretically as the quotient space of $\tilde{\mathcal{E}}$ by this action:

$$
\mathcal{E}:=\tilde{\mathcal{E}} / C_{N}^{2}
$$

The canonical projection to $\Delta$ is denoted by $\pi: \mathcal{E} \rightarrow \Delta$. The fibre $\pi^{-1}(0)$ is the singular curve with one ordinary double point.

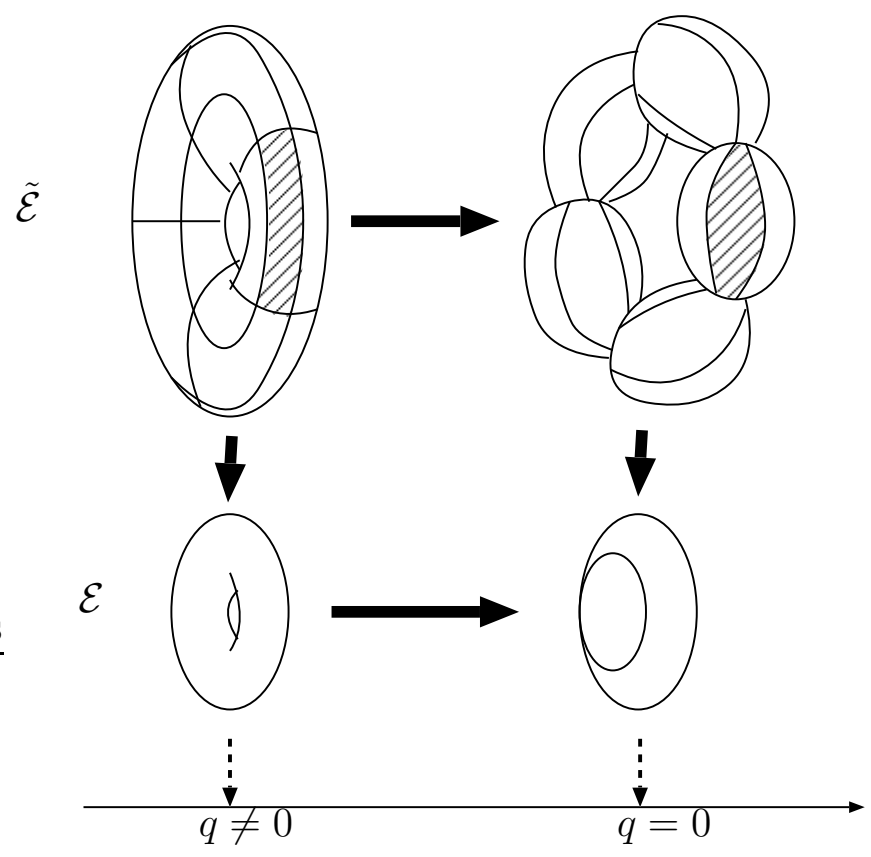

Figure 1. Degeneration of an elliptic curve and its $N^{2}$-covering.

The family of $L$-pointed elliptic curves and its covering are defined by

$$
\mathfrak{X}:=\mathcal{E} \times_{\Delta} S, \quad \tilde{\mathfrak{X}}:=\tilde{\mathcal{E}} \times_{\Delta} S .
$$


Here the base space $S$ is the fibre product of $\mathcal{E}$ 's without diagonals:

$$
\begin{aligned}
S:= & \{\left(q ; Q_{1}, \ldots, Q_{L}\right) \in \overbrace{\mathcal{E} \times_{\Delta} \cdots \times_{\Delta} \mathcal{E}}^{L} \\
& \left.\mid Q_{i} \neq Q_{j}(i \neq j), Q_{i} \neq\left[(0,0,0)_{k}\right] \text { for any k. }\right\},
\end{aligned}
$$

where $Q_{i}$ is the point of the $i$-th $\mathcal{E}$ in the fibre product with $\pi\left(Q_{i}\right)=q$. We exclude the degeneration of the types $Q_{i} \rightarrow Q_{j}$ and $Q_{i} \rightarrow$ (node). We denote the canonical projections $\mathfrak{X} \rightarrow S, \tilde{\mathfrak{X}} \rightarrow S$ and $\tilde{\mathfrak{X}} \rightarrow \mathfrak{X}$ by $\pi_{\mathfrak{X} / S}, \pi_{\tilde{\mathfrak{X}} / S}$ and $\pi_{\tilde{\mathfrak{X}} / \mathfrak{X}}$ respectively. The fibres of $\tilde{\mathfrak{X}}$ and $\mathfrak{X}$ over $S_{0}:=$ $\left\{\left(0 ; Q_{1}, \ldots, Q_{L}\right) \in S\right\}$ are singular curves.

The section $q_{i}$ of $\mathfrak{X} \rightarrow S$ is defined by

$$
q_{i}\left(\left(q ; Q_{1}, \ldots, Q_{L}\right)\right):=\left(q ; Q_{i} ; Q_{1}, \ldots, Q_{L}\right) .
$$

We denote the divisor $\left[q_{1}(S)\right]+\cdots+\left[q_{L}(S)\right]$ by $D$.

The group $C_{N}^{2}$ acts on $\tilde{\mathfrak{X}}$ naturally as covering transformation and on $\mathfrak{g}$ by

$$
(m, n) \cdot A=\left(\gamma^{m} \beta^{n}\right) A\left(\gamma^{m} \beta^{n}\right)^{-1}
$$

The twisted Lie algebra bundle $\mathfrak{g}_{\mathfrak{X}}^{\text {tw }}$ on $\stackrel{\circ}{\mathfrak{X}}:=\mathfrak{X} \backslash\{$ singular points $\}$ is defined as the associated bundle to the $C_{N}^{2}$-principal bundle $\stackrel{\circ}{\tilde{\mathfrak{X}}}:=$ $\tilde{\mathfrak{X}} \backslash\{$ singular points $\} \rightarrow \stackrel{\circ}{\mathfrak{X}}:$

$$
\mathfrak{g}_{\mathfrak{X}}^{\mathrm{tw}}:=\stackrel{\circ}{\tilde{\mathfrak{X}}} \times_{C_{N}^{2}} \mathfrak{g} .
$$

It is obvious that the restriction of $\mathfrak{g}_{\mathfrak{X}}^{\text {tw }}$ to a fibre of $\mathfrak{X}$ at a point $\left(q ; Q_{1}, \ldots, Q_{L}\right)(q \neq 0)$ is the bundle $\mathfrak{g}^{\mathrm{tw}}$ on the elliptic curve $\mathbb{C}^{\times} / q^{\mathbb{Z}}$ defined by (3).

Sheaf version of affine Lie algebras $\mathfrak{g}^{P}, \mathfrak{g}_{+}^{P}, \mathfrak{g}^{D}$ and $\hat{\mathfrak{g}}^{D}$ (cf. (7)), (8), (10) ) are $\mathcal{O}_{S^{-}}$Lie algebras defined by

$$
\begin{array}{ll}
\mathfrak{g}_{S}^{Q_{i}}:=\pi_{\mathfrak{X} / S, *}\left(\mathfrak{g}_{\mathfrak{X}}^{\mathrm{tw}}\left(* Q_{i}\right)\right)_{Q_{i}}^{\wedge}, & \mathfrak{g}_{S,+}^{Q_{i}}:=\pi_{\mathfrak{X} / S, *}\left(\mathfrak{g}_{\mathfrak{X}}^{\mathrm{tw}}\right)_{Q_{i}}^{\wedge}, \\
\mathfrak{g}_{S}^{D}:=\pi_{\mathfrak{X} / S, *}\left(\mathfrak{g}_{\mathfrak{X}}^{\mathrm{tw}}(* D)\right)_{D}^{\wedge}=\bigoplus_{i=1}^{L} \mathfrak{g}_{S}^{Q_{i}}, & \mathfrak{g}_{S,+}^{D}:=\pi_{\mathfrak{X} / S, *}\left(\mathfrak{g}_{\mathfrak{X}}^{\mathrm{tw}}\right)_{D}^{\wedge}=\bigoplus_{i=1}^{L} \mathfrak{g}_{S,+}^{Q_{i}} .
\end{array}
$$

Since we assume that sections $Q_{i}$ do not touch the singular point of the singular fibre, the definitions are the same as those for the non-singular case, (3.11) of [KT]. The central extensions of $\mathfrak{g}_{S}^{D}$ and $\mathfrak{g}_{S,+}^{D}$ are defined by the cocycle (9) with the coefficients in $\mathcal{O}_{S}$ :

$$
\hat{\mathfrak{g}}_{S}^{D}:=\mathfrak{g}_{S}^{D} \oplus \mathcal{O}_{S} \hat{k}, \quad \hat{\mathfrak{g}}_{S,+}^{D}:=\mathfrak{g}_{S,+}^{D} \oplus \mathcal{O}_{S} \hat{k} .
$$


The Lie subalgebra of meromorphic sections $\mathfrak{g}_{\text {out }} \subset \hat{\mathfrak{g}}^{D}$, (12), would be replaced by $\pi_{\mathfrak{X} / S, *}\left(\mathfrak{g}_{\mathfrak{X}}^{\mathrm{tw}}(* D)\right)$ if there were no singularity, as was the case in $[\mathrm{KT}]$. Taking the singular fibre into account, we modify this naive definition as follows:

$$
\mathfrak{g}_{\mathfrak{X}, \text { out }}=\left(\pi_{\tilde{\mathfrak{X}} / S, *}\left(\mathfrak{g} \otimes \mathcal{O}_{\tilde{\mathfrak{X}}}(* \tilde{D})\right)\right)^{C_{N}^{2}},
$$

where $\tilde{D}$ is the $C_{N}^{2}$-orbit of the divisor $D$ and $(\cdot)^{C_{N}^{2}}$ denotes the $C_{N^{-}}^{2}$ invariant section of the equivariant locally free sheaf $\mathfrak{g} \otimes \mathcal{O}_{\tilde{\mathfrak{x}}}$. In other words, a $\mathfrak{g}$-valued meromorphic function $f(s, P)\left(s=\left(q ; Q_{1}, \ldots, Q_{L}\right) \in\right.$ $\left.S,\left.P \in \tilde{\mathcal{E}}\right|_{s}\right)$ belongs to $\mathfrak{g}_{\mathfrak{x} \text {,out }}$ if and only if it satisfies

$$
f(s,(1,0) \cdot P)=\operatorname{Ad} \gamma(f(s, P)), \quad f(s,(0,1) \cdot P)=\operatorname{Ad} \beta(f(s, P)),
$$

where $(m, n) \cdot(m, n \in \mathbb{Z})$ is the left action of the generators of $C_{N}^{2}$ on the fibre of $\tilde{\mathcal{E}}$ defined by $(m, n) \cdot P:=P \cdot(-m,-n)$. (See (17).)

Our construction is so explicit that we have an explicit basis of $\mathfrak{g}_{\mathfrak{X} \text {,out }}$. In $[\mathrm{KT}]$ meromorphic functions $w_{a b}(\tau ; t)(a, b=0, \ldots, N-1, \operatorname{Im} \tau>0$, $t \in \mathbb{C}$ ) characterised by the following properties were introduced:

- Additive quasi-periodicity: $w_{a b}(\tau ; t+1)=\varepsilon^{a} w_{a b}(\tau ; t), w_{a b}(\tau ; t+$ $\tau)=\varepsilon^{b} w_{a b}(\tau ; t)$

- As a function of $t \in \mathbb{C}, w_{a b}(\tau ; t)$ has a simple pole with residue 1 at $\mathbb{Z}+\mathbb{Z} \tau$.

Let us denote this function by $w_{a b}^{\text {add }}(\tau ; t)$. (The superscript "add" stands for "additive".) Let us rewrite it to a multiplicatively quasiperiodic function $w_{a b}^{\operatorname{mul}}(q ; u)$ as follows:

$$
\begin{aligned}
& w_{a b}^{\mathrm{mul}}(q ; u):=\frac{2 \pi i u^{a}}{u^{N}-1} \times \\
& \quad \times \frac{\left(q^{N-a} \varepsilon^{b} u^{-N} ; q^{N}\right)_{\infty}\left(q^{a} \varepsilon^{-b} u^{N} ; q^{N}\right)_{\infty}}{\left(q^{N-a} \varepsilon^{b} ; q^{N}\right)_{\infty}\left(q^{N} u^{-n} ; q^{N}\right)_{\infty}\left(q^{a} \varepsilon^{-b} ; q^{N}\right)_{\infty}\left(q^{N} u^{N} ; q^{N}\right)_{\infty}},
\end{aligned}
$$

where $(x ; q)_{\infty}=\prod_{n=0}^{\infty}\left(1-x q^{n}\right)$ is the standard infinite product symbol. The function $w_{a b}^{\mathrm{mul}}(q ; u)$ is related to $w_{a b}^{\mathrm{add}}$ by $w_{a b}^{\mathrm{mul}}(q ; u)=w_{a b}^{\mathrm{add}}(N \log u / 2 \pi i, N \log q / 2 \pi i)$ when $q \neq 0$, that is, we replaced the arguments of $w_{a b}^{\text {add }}$ by $e^{2 \pi i z}=u^{N}$, $e^{2 \pi i \tau}=q^{N}$ and used the product formula for the theta function. When $q=0, w_{a b}^{\text {mul }}$ becomes a rational function of $u$ :

$$
w_{a b}^{\mathrm{mul}}(0 ; u)= \begin{cases}2 \pi i u^{a}\left(u^{N}-1\right)^{-1}, & (a \neq 0), \\ 2 \pi i\left(1-\varepsilon^{b}\right)^{-1}\left(u^{N}-\varepsilon^{b}\right)\left(u^{N}-1\right)^{-1}, & (a=0) .\end{cases}
$$

The important property of $w_{a b}^{\mathrm{mul}}$ is that it inherits the quasi-periodicity of $w_{a b}^{\text {add }}$ :

$$
w_{a b}^{\mathrm{mul}}(q ; \varepsilon u)=\varepsilon^{a} w_{a b}^{\mathrm{mul}}(q ; u), \quad w_{a b}^{\mathrm{mul}}(q ; q u)=\varepsilon^{b} w_{a b}^{\mathrm{mul}}(q ; u) .
$$


We define the function $w_{a b, i}(P)(i=1, \ldots, L)$ on $\tilde{\mathfrak{X}}$ in terms of $w_{a b}^{\text {mul }}$ : for $\left(q, x_{k}, y_{k}\right)_{k} \in U_{k}$ (cf. (14) $), q \neq 0$,

$$
w_{a b, i}\left(\left(\left(q, x_{k}, y_{k}\right)_{k}, s\right)\right):=w_{a b}^{\mathrm{mul}}\left(q ; q^{k-k^{\prime}} x_{k} / x_{k^{\prime}}^{\prime}\right) .
$$

Here $s=\left(q ; Q_{1}, \ldots, Q_{L}\right) \in S$ and we fix an index $k^{\prime}$ to express $Q_{i}$ as a point $\left(q, x_{k^{\prime}}^{\prime}, y_{k^{\prime}}^{\prime}\right)_{k^{\prime}}$ in $U_{k^{\prime}}$. (The function $w_{a b, i}$ is determined up to this choice.) This function is extended to the points with $q=0$. (For example, $w_{a b, i}\left(\left(0, x_{0}, y_{0}\right)_{0}\right)$ is the rational function (29) of $x=$ $x_{0} / x_{0}^{\prime}$ when $Q_{i}$ is represented as a point $\left(0 ; x_{0}^{\prime}, y_{0}^{\prime}\right)_{0}$ in $U_{0}$.) The main properties of this function are

- Quasi-periodicity with respect to $C_{N}^{2}$-action:

$$
w_{a b, i}((1,0) \cdot P)=\varepsilon^{a} w_{a b, i}(P), \quad w_{a b, i}((0,1) \cdot P)=\varepsilon^{b} w_{a b, i}(P),
$$

- All poles are simple and located at $Q_{i}$ modulo $C_{N}^{2}$ action.

It is easy to see that any section of $\mathfrak{g}_{\mathfrak{X} \text {,out }}$ is a linear combination of $J_{a b} \otimes w_{a b, i}(P)$ 's and their derivatives along the fibre.

Lemma 3.1. $\hat{\mathfrak{g}}_{S}^{D}=\mathfrak{g}_{\mathfrak{X}, \text { out }} \oplus \hat{\mathfrak{g}}_{S,+}^{D}$.

Proof. The singular part of an element of $\hat{\mathfrak{g}}_{S}^{D}$ can be expressed by a linear combination of $J_{a b} \otimes w_{a b, i}(P)$ and derivatives in a unique way. Subtracting such combination which belongs to $\mathfrak{g}_{\mathfrak{X} \text {,out }}$, we end up with a regular element of $\hat{\mathfrak{g}}_{S,+}^{D}$.

\section{Sheaves of CONFORMAL COINVARIANTS AND CONFORMAL BLOCKS}

In this section we introduce the sheaf $\mathcal{C C}$ of conformal coinvariants and the sheaf $\mathcal{C B}$ of conformal blocks and show their basic properties.

Definitions of $\mathcal{C C}$ and $\mathcal{C B}$ are literally the same as those for the nonsingular case, Definition 3.3 of [KT].

Definition 4.1. For any $\hat{\mathfrak{g}}_{S}^{D}$-module $\mathcal{M}$ of level $k$ (i.e., $\hat{k}$ acts as $k \cdot \mathrm{id}$ ), we define the sheaf $\mathcal{C C}(\mathcal{M})$ of conformal coinvariants and the sheaf $\mathcal{C B}(\mathcal{M})$ of conformal blocks by

$$
\begin{aligned}
\mathcal{C C}(\mathcal{M}) & :=\mathcal{M} / \mathfrak{g}_{\mathfrak{X}, \text { out }} \mathcal{M} \\
\mathcal{C B}(\mathcal{M}) & :=\mathcal{H} \text { om }_{\mathcal{O}_{S}}\left(\mathcal{C C}(\mathcal{M}), \mathcal{O}_{S}\right) .
\end{aligned}
$$

We can regard $\mathcal{C C}(\cdot)$ as a covariant right exact functor from the category of $\hat{\mathfrak{g}}_{S}^{D}$-modules to that of $\mathcal{O}_{S}$-modules and similarly $\mathcal{C B}(\cdot)$ as a contravariant left exact functor. 
The goal of this paper is to prove that $\mathcal{C C}$ and $\mathcal{C B}$ are locally free. Since $\mathcal{C B}$ is the dual of $\mathcal{C C}$, we mainly discuss about $\mathcal{C C}$ and briefly mention on $\mathcal{C B}$ when it is necessary.

We assume that the $\hat{\mathfrak{g}}_{S}^{D}$-module $\mathcal{M}$ are of the following type:

$$
\mathcal{M}=\mathcal{O}_{S} \otimes \bigotimes_{i=1}^{L} M_{i}=\bigotimes_{i=1}^{L}\left(\mathcal{O}_{S} \otimes M_{i}\right),
$$

where each $M_{i}$ is a quotient of a $\hat{\mathfrak{g}}$-Weyl module $M\left(V_{i}\right):=\operatorname{Ind}_{\hat{\mathfrak{g}}_{+}^{Q_{i}}}^{\mathfrak{g}^{Q_{i}} \oplus \mathbb{C} \hat{k}} V_{i}$ of level $k$ for a finite dimensional irreducible $\mathfrak{g}$-module $V_{i}$. (See $\S 6$ of [T] or $\S 2.4$ of $\left[\mathrm{KL}\right.$.) To endow $\mathcal{M}$ in (35) with the $\hat{\mathfrak{g}}_{S}^{D}$-module structure, we need to fix the coordinate of $\mathcal{E}$ and the trivialisation of $\mathfrak{g}_{\mathfrak{X}}^{\text {tw }}$, which is irrelevant to the statements of theorems below. In the concrete computations, we use the coordinates and the trivialisation obtained naturally from the construction in $\$ 3$.

Proposition 4.2. $\mathcal{C C}(\mathcal{M})$ is a coherent $\mathcal{O}_{S}$-sheaf. When all $M_{i}$ 's are Weyl modules, $\mathcal{C C}(\mathcal{M})$ is (and hence $\mathcal{C B}(\mathcal{M})$ is) locally free.

Proof. Lemma 3.1 makes it possible to apply the same argument as the proof for the non-singular case, Corollary 3.5 of $[\mathrm{KT}]$. In fact, if each $M_{i}$ in (35) is a Weyl module $M\left(V_{i}\right), \mathcal{M}$ is expressed as

$$
\mathcal{M}=U_{S}\left(\hat{\mathfrak{g}}_{S}^{D}\right) \otimes_{U_{S}\left(\hat{\mathfrak{g}}_{S,+}^{D}\right)}\left(V \otimes \mathcal{O}_{S}\right) \leftarrow U_{S}\left(\mathfrak{g}_{\mathfrak{X}, \text { out }}\right) \otimes_{\mathcal{O}_{S}}\left(V \otimes \mathcal{O}_{S}\right),
$$

by the Poincaré-Birkhoff-Witt theorem $\left(V=\otimes_{i=1}^{L} V_{i}\right)$. Here $U_{S}(\cdot)$ denotes the universal $\mathcal{O}_{S}$-enveloping algebra. Modding out by $\mathfrak{g}_{\mathfrak{x} \text {,out }} \mathcal{M}$, we have

$$
\mathcal{C C}(\mathcal{M})=\mathcal{M} / \mathfrak{g}_{\mathfrak{X} \text {,out }} \mathcal{M} \stackrel{\sim}{\leftarrow} V \otimes \mathcal{O}_{S},
$$

which means that $\mathcal{C C}(\mathcal{M})$ is locally free, in particular, coherent.

If $M_{i}$ 's are quotients of Weyl modules, $\mathcal{M}$ is a quotient of $\otimes M\left(V_{i}\right) \otimes$ $\mathcal{O}_{S}$. Hence by the right exactness of the functor $\mathcal{C C}, \mathcal{C C}(\mathcal{M})$ is a quotient of a coherent sheaf, and therefore coherent.

In $\$ 6$ we prove the locally freeness of $\mathcal{C C}(\mathcal{M})$ for integrable $M_{i}$ 's, examining the behaviour of $\mathcal{C C}(\mathcal{M})$ at the boundary of the moduli space $\left(S_{0}=\{q=0\} \subset S\right)$ carefully.

\section{SheAf VERSION OF TRIGONOMETRIC AND ORBIFOLD WZW MODEL}

Everything in previous two sections can be restricted on $S_{0}$, namely on the configuration space of points on a singular rational curve with one ordinary double point. (As we have mentioned, the restriction of 
the functions $w_{a b, i}$ needs special care.) Hence we can define the corresponding sheaves $\mathcal{C C}(\mathcal{M})$ and $\mathcal{C B}(\mathcal{M})$ which we denote by $\mathcal{C C}_{\text {trig }}(\mathcal{M})$ and $\mathcal{C B}_{\text {trig }}(\mathcal{M})$. The subscript "trig" is put here because, as we shall see below, there are connections on them expressed in terms of the trigonometric $r$ matrix.

In the proof of Theorem 6.1 we shall use the sheaf of conformal coinvariants of the orbifold WZW model, $\mathcal{C C}_{\text {orb }}\left(\mathcal{M}_{0} \otimes \mathcal{M} \otimes \mathcal{M}_{\infty}\right)$ on $S_{0}$, where $\mathcal{M}_{*}=M_{*} \otimes \mathcal{O}_{S_{0}}$ for a $\hat{\mathfrak{g}}^{(*)}$-module $M_{*}(*=0, \infty)$. We shall recall the definition of the twisted affine algebras $\hat{\mathfrak{g}}^{(0)}$ and $\hat{\mathfrak{g}}^{(\infty)}$ and the details of $\mathrm{CC}_{\text {orb }}$ soon later. Here we only say that $\mathcal{C} \mathcal{C}_{\text {orb }}$ is defined exactly in the same way as $\mathcal{C C}_{\text {trig }}(\mathcal{M})$ if we replace the degenerate elliptic curve (the fibre of $\mathcal{E}$ at $q=0$ ) by the orbifold $\mathbb{P}^{1} / C_{N}$. Note that $S_{0}$ can be regarded as the configuration space of points on $\mathbb{P}^{1} / C_{N}$. (See $\S 3$ of [T].)

Let $\mathcal{M}$ be as in (35) and $M_{*}$ be a $\hat{\mathfrak{g}}^{(*)}$-Verma module. Then Proposition 4.2 holds for $\mathcal{C C}_{\text {trig }}, \mathcal{C B}_{\text {trig }}, \mathcal{C C}_{\text {orb }}$ and $\mathcal{C B}_{\text {orb }}$ as well. In fact, we can prove locally freeness under this assumption.

Proposition 5.1. (i) The sheaf $\mathcal{C C}_{\text {trig }}(\mathcal{M})$ and the sheaf $\mathcal{C} \mathcal{B}_{\text {trig }}(\mathcal{M})$ are locally free $\mathcal{O}_{S_{0}}$-sheaves.

(ii) The sheaf $\mathcal{C C}_{\text {orb }}\left(\mathcal{M}_{0} \otimes \mathcal{M} \otimes \mathcal{M}_{\infty}\right)$ and the sheaf $\mathcal{C B}_{\text {orb }}\left(\mathcal{M}_{0} \otimes\right.$ $\left.\mathcal{M} \otimes \mathcal{M}_{\infty}\right)$ are locally free $\mathcal{O}_{S_{0}}$-sheaves.

Proof. (i) The proof of the locally freeness for the non-singular case, Corollary 5.3 of $[\mathrm{KT}]$ is true also in this case: $\mathcal{C C}_{\text {trig }}(\mathcal{M})$ is coherent as shown at the end of $\$ 4$ and there is a connection and $D$-module structure on it, which implies that it is locally free $\mathcal{O}_{S_{0}}$-sheaf. The only difference is that we do not change the curve itself (the modulus $q$ is fixed to 0 ) in the present case, and hence there is nothing corresponding to the connection in the direction of $\partial / \partial \tau$ in $[\mathbf{K T}$. The connection in the direction of $\partial / \partial z_{i}\left(z_{i}\right.$ is the coordinate of $\left.Q_{i}\right)$ is $\nabla_{i}=\partial / \partial z_{i}-\rho_{i}(T[-1])$ (cf. (5.14) of [KT]) as is well-known, where $\rho_{i}$ is the representation of the Virasoro algebra on $M_{i}$ constructed via the Sugawara construction and $T[-1]$ is one of the Virasoro generator, usually denoted by $L_{-1}$.

(ii) We might proceed as the proof of (i) from the beginning but the short cut is to use the result of (i). The coherence of $\mathcal{C} \mathcal{C}_{\text {orb }}\left(\mathcal{M}_{0} \otimes\right.$ $\left.\mathcal{M} \otimes \mathcal{M}_{\infty}\right)$ having been proved, we have only to check that the above connection operators $\nabla_{i}(i=1, \ldots, L)$ on $\mathcal{C C}_{\text {trig }}(\mathcal{M})$ also define the flat connection on $\mathcal{C C}_{\text {orb }}$. What we need to check is

- $\left[\nabla_{i}, \mathfrak{g}_{\text {out }}^{\text {orb }}\right] \subset \mathfrak{g}_{\text {out }}^{\text {orb }}$

- $\left[\nabla_{i}, \nabla_{j}\right]=0$ on $\mathcal{C C}_{\text {orb }}$, 
which is proved in the same way as in the case of the ordinary WZW model, e.g., Lemma 4 of [FFR].

The connection on $\mathcal{C} \mathcal{C}_{\text {trig }}(\mathcal{M})$ mentioned in the proof of (i) is obtained by the degeneration $q \rightarrow 0$ of the elliptic Knizhnik-Zamolodchikov connection in $[\mathrm{E}$ and $[\mathrm{KT}$. They are expressed as the first order differential operators on $V \otimes \mathcal{O}_{S_{0}}$ in terms of the trigonometric $r$ matrix. In fact, by tracing the argument which leads to the explicit form (Theorem 5.9 in $[\mathrm{KT}]$ ) of the connection, we have only to replace the functions $w_{a b}\left(z_{j}-z_{i}\right)$ there with $w_{a b, i}\left(Q_{j}\right)$ which is expressed by the rational function of the form (29) on $S_{0}$. Hence the KZ equation for the WZW model on the degenerate elliptic curve is the trigonometric $K Z$ equation.

Lemma 5.2. (i) The fibre of $\mathcal{C C}_{\text {trig }}(\mathcal{M})$ at $s \in S_{0},\left.\mathcal{C C}_{\text {trig }}(\mathcal{M})\right|_{s}$, is isomorphic to $\mathrm{CC}_{\text {trig }}(M)$, the space of conformal coinvariants of the trigonometric WZW model for the geometric data corresponding to s.

(ii) The fibre of $\mathcal{C C}_{\text {orb }}(\mathcal{M})$ at $s,\left.\mathcal{C C}_{\text {orb }}(\mathcal{M})\right|_{s}$, is isomorphic to $\mathrm{CC}_{\text {orb }}(M)$, the space of conformal coinvariants of the orbifold $W Z W$ model for the geometric data corresponding to $s$.

See Definition 3.2 of [T] for the definition of $\mathrm{CC}_{\text {trig }}$ and $\mathrm{CC}_{\text {orb }}$.

Proof. We can modify the proof of the corresponding statement for the non-singular case, Corollary 5.4 of $[\mathrm{KT}]$. For example, for the case (i), the isomorphism $\left.\mathfrak{g}_{\mathfrak{X} \text {,out }}\right|_{s} \cong \mathfrak{g}_{\text {out }}^{\text {trig }}$ is a consequence of the existence of the sections $w_{a b, i}$ and their derivatives (cf. the end of \$3) which span both $\left.\mathfrak{g}_{\mathfrak{X} \text {,out }}\right|_{s}$ and $\mathfrak{g}_{\text {out }}^{\text {trig }}$. The rest of the proof can be translated to the present case without change.

The proof of (ii) is similar.

Combining Proposition 5.1 Lemma 5.2 and Theorem 5.1 of $[\mathrm{T}]$, we have the following isomorphism:

$$
\iota: \mathcal{C} \mathcal{C}_{\text {trig }}(\mathcal{M}) \stackrel{\sim}{\rightarrow} \bigoplus_{\lambda \in \operatorname{wt}(V)} \mathcal{C} \mathcal{C}_{\text {orb }}\left(\mathcal{M}_{\tilde{\lambda}}^{(0)} \otimes \mathcal{M} \otimes \mathcal{M}_{\tilde{\lambda}^{\prime}}^{(\infty)}\right)
$$

where $V=\bigotimes_{i=1}^{L} V_{i}$ is the $\mathfrak{g}$-module generating $M=\bigotimes_{i=1}^{L} M_{i}$ (recall $M_{i}$ is a quotient of Weyl module $\left.M\left(V_{i}\right)\right)$, wt $(V)$ is the set of its weights,

$$
\begin{aligned}
& \tilde{\lambda}:=-\lambda \circ\left(1-\operatorname{Ad} \beta^{-1}\right)^{-1}=\lambda \circ(1-\operatorname{Ad} \beta)^{-1} \circ \operatorname{Ad} \beta, \\
& \tilde{\lambda}^{\prime}:=-\lambda \circ(1-\operatorname{Ad} \beta)^{-1},
\end{aligned}
$$

$\mathcal{M}_{\mu}^{(*)}=M_{\mu}^{(*)} \otimes \mathcal{O}_{S_{0}}$ for a Verma module $M_{\mu}^{(*)}$ of $\hat{\mathfrak{g}}^{(*)}$ with the highest weight $\mu$ (cf. Definition 4.1 (i) of [T] ). 
In 96 the modules $M_{i}$ are assumed to be integrable highest weight modules. (cf. Chapter 10 of $[\mathrm{K}$.) In this case the above result can be refined. For this purpose we recall the details of the orbifold WZW model defined in $\S 3$ of $[\mathrm{T}]$.

Let us denote the standard coordinate of $\mathbb{P}^{1}(\mathbb{C})$ by $t$. The cyclic group $C_{N}$ acts as $t \mapsto \varepsilon^{a} t\left(a \in C_{N}\right)$ and the quotient $E_{\text {orb }}=\mathbb{P}^{1} / C_{N}$ is an ordinary orbifold.

The definition of the space of conformal coinvariants/blocks of the orbifold WZW model on $E_{\text {orb }}$ is almost the same as that on elliptic curves, Definition 2.1, except that we also insert modules to the singular points 0 and $\infty$. The Lie algebra $\mathfrak{g}_{\text {out }}$ in (13) is replaced by $\mathfrak{g}_{\text {out }}^{\text {orb }}$ which consists of $\mathfrak{g}$-valued meromorphic functions $f(t)$ on $\mathbb{P}^{1}$ such that: (1) poles belong to $\left\{0, Q_{1}, \ldots, Q_{L}, \infty\right\} ;(2) f(\varepsilon t)=\operatorname{Ad} \gamma(f(t))$. Accordingly, the module inserted at 0 is the $\hat{\mathfrak{g}}^{(0)}$-module and the module inserted at $\infty$ is the $\hat{\mathfrak{g}}^{(\infty)}$-module, where

$$
\begin{aligned}
\hat{\mathfrak{g}}^{(0)} & =\bigoplus_{\substack{a, b=0, \ldots, N-1 \\
(a, b) \neq(0,0)}} \bigoplus_{m \in \mathbb{Z}} \mathbb{C} J_{a, b} \otimes t^{a+m N} \oplus \mathbb{C} \hat{k}, \\
\mathfrak{g}^{(\infty)} & =\bigoplus_{\substack{a, b=0, \ldots, N-1 \\
(a, b) \neq(0,0)}} \bigoplus_{m \in \mathbb{Z}} \mathbb{C} J_{a, b} \otimes t^{a+m N} \oplus \mathbb{C} \hat{k} \\
& =\bigoplus_{\substack{a, b=0, \ldots, N-1 \\
(a, b) \neq(0,0)}} \bigoplus_{m \in \mathbb{Z}} \mathbb{C} J_{a, b} \otimes s^{-a+m N} \oplus \mathbb{C} \hat{k} . \quad\left(s=t^{-1}\right)
\end{aligned}
$$

The cocycles which defines the central extension of $\mathfrak{g}^{(0)}$ and $\mathfrak{g}^{(\infty)}$ are:

$$
\mathrm{c}_{\mathrm{a}, 0}(A, B):=\frac{1}{N} \operatorname{Res}_{t=0}(d A \mid B), \quad \mathrm{c}_{\mathrm{a}, \infty}(A, B):=\frac{1}{N} \operatorname{Res}_{s=0}(d A \mid B),
$$

for $A, B \in \mathfrak{g}^{(0)}$ and $A, B \in \mathfrak{g}^{(\infty)}$ respectively. ( $\mathfrak{g}^{(*)}$ is the loop algebra part of $\hat{\mathfrak{g}}^{(*)}$.)

As Etingof showed (Lemma 1.1 of $[\mathrm{E}]$ ), $\hat{\mathfrak{g}}^{(0)}$ and $\hat{\mathfrak{g}}^{(\infty)}$ are isomorphic to the ordinary affine Lie algebra $\hat{\mathfrak{g}}$ of $A_{N-1}^{(1)}$ type. Explicitly the isomorphism $\phi_{0}: \hat{\mathfrak{g}}^{(0)} \stackrel{\sim}{\rightarrow} \hat{\mathfrak{g}}$ is defined by

$$
\begin{gathered}
\phi_{0}\left(E_{i, i+1} \otimes t\right)=e_{i}, \quad \phi_{0}\left(E_{i+1, i} \otimes t^{-1}\right)=f_{i}, \\
\phi_{0}\left(E_{N, 1} \otimes t\right)=e_{0}, \quad \phi_{0}\left(E_{1, N} \otimes t^{-1}\right)=f_{0}, \\
\phi_{0}\left(H_{i, i+1} \otimes 1\right)=\alpha_{i}^{\vee}-\frac{\hat{k}}{N}, \quad \phi_{0}(\hat{k})=\hat{k},
\end{gathered}
$$

for $i=1, \ldots, N-1$, where $E_{i j}$ is the matrix unit, $H_{i j}=E_{i i}-E_{j j}$, $e_{i}, f_{i}(i=0, \ldots, N-1)$ are the Chevalley generators of $\hat{\mathfrak{g}}$ and $\alpha_{i}^{\vee}$ 
$(i=0, \ldots, N-1)$ are coroots of $\hat{\mathfrak{g}}$. (cf. $\S 6.2$ and $\S 7.4$ of $[\mathrm{K}]$.) Since the positive powers of $s$ (cf. (41)) kill the highest weight vector of the Verma module $M_{\mu}^{(\infty)}$ inserted at $\infty$ (cf. Definition 4.1 (i) of [T]), we identify $\hat{\mathfrak{g}}^{(\infty)}$ and $\hat{\mathfrak{g}}$ through an isomorphism which is essentially a composition of $\phi_{0}$ and the Chevalley involution:

$$
\begin{aligned}
\phi_{\infty}\left(E_{i, i+1} \otimes s^{-1}\right) & =-f_{i}, \quad \phi_{\infty}\left(E_{i+1, i} \otimes s\right)=-e_{i}, \\
\phi_{\infty}\left(E_{N, 1} \otimes s^{-1}\right) & =-f_{0}, \quad \phi_{\infty}\left(E_{1, N} \otimes s\right)=-e_{0}, \\
\phi_{\infty}\left(H_{i, i+1} \otimes 1\right) & =-\alpha_{i}^{\vee}+\frac{\hat{k}}{N}, \quad \phi_{\infty}(\hat{k})=\hat{k},
\end{aligned}
$$

for $i=1, \ldots, N-1$. Identified through $\phi_{0}$ and $\phi_{\infty}$, the Verma modules of $\hat{\mathfrak{g}}^{(0)}$ and $\hat{\mathfrak{g}}^{(\infty)}$ are Verma modules of $\hat{\mathfrak{g}}$ in ordinary sense.

In this section, $e_{i}$ and $f_{i}$ denote the Chevalley generators of $\hat{\mathfrak{g}}$ identified with the elements in $\hat{\mathfrak{g}}^{(0)}$ or $\hat{\mathfrak{g}}^{(\infty)}$ by means of $\phi_{0}$ or $\phi_{\infty}$.

Proposition 5.3. Assume that all $M_{i}(i=1, \ldots, L)$ are integrable highest weight modules and that $M_{\mu_{*}}^{(*)}(*=0, \infty)$ is a Verma modules of $\hat{\mathfrak{g}}^{(*)}$.

Then $\mathrm{CC}_{\text {orb }}\left(M_{\mu_{0}}^{(0)} \otimes M \otimes M_{\mu_{\infty}}^{(\infty)}\right)$ is 0 unless $\mu_{0}$ and $\mu_{\infty}$ are dominant integral weights of $\hat{\mathfrak{g}}$ identified with $\hat{\mathfrak{g}}^{(0)}$ and $\hat{\mathfrak{g}}^{(\infty)}$. If $\mu_{0}$ and $\mu_{\infty}$ are dominant integral weights,

$$
\mathrm{CC}_{\text {orb }}\left(M_{\mu_{0}}^{(0)} \otimes M \otimes M_{\mu_{\infty}}^{(\infty)}\right) \cong \mathrm{CC}_{\text {orb }}\left(L_{\mu_{0}}^{(0)} \otimes M \otimes L_{\mu_{\infty}}^{(\infty)}\right),
$$

where $L_{\mu_{*}}^{(*)}(*=0, \infty)$ is the irreducible quotient of $M_{\mu_{*}}^{(*)}$.

Remark 5.4. In physics context, this proposition is a consequence of the propagation of the null field. See $\S 4$ of $[\mathrm{Z}$. The author thanks Yasuhiko Yamada for this comment.

Remark 5.5. Proposition 5.3 is in sharp contrast to the Weyl module case. See $\S 6$ of [T].

Proof. The following lemma shall be proved later.

Lemma 5.6. Let $N_{*}(*=0, \infty)$ be a quotient of the Verma module $M_{\mu_{*}}^{(*)}$. Suppose $v_{\kappa} \in N_{0}$ is a singular vector of weight $\kappa$ which is not a dominant integral weight. (The weight $\kappa$ may possibly be the highest weight $\mu_{0}$.) Then for any $v \in M$ and $v_{\infty} \in N_{\infty}$,

$$
v_{\kappa} \otimes v \otimes v_{\infty} \equiv 0 \quad \bmod \mathfrak{g}_{\text {out }}^{\text {orb }}\left(N_{0} \otimes M \otimes N_{\infty}\right) .
$$

The same is true for a singular vector $v_{\kappa} \in N_{\infty}$. 
The first statement of Proposition 5.3 is a consequence of Lemma 5.6. For example, assume $\mu_{0}$ is not a dominant integral weight. Let us show

$$
X_{1}\left[-n_{1}\right] \cdots X_{l}\left[-n_{l}\right]\left|\mu_{0}\right\rangle \otimes v \otimes v_{\infty} \equiv 0 \quad \bmod \mathfrak{g}_{\text {out }}^{\text {orb }}
$$

where $X_{i}\left[-n_{i}\right] \in \hat{\mathfrak{g}}^{(0)}\left(X_{i} \in \mathfrak{g}, n_{i}>0\right), v \in M, v_{\infty} \in M_{\mu_{\infty}}^{(\infty)}$ and $\bmod \mathfrak{g}_{\text {out }}^{\text {orb }}$ denotes $\bmod \mathfrak{g}_{\text {out }}^{\text {orb }}\left(M_{\mu_{0}}^{(0)} \otimes M \otimes M_{\mu_{\infty}}^{(\infty)}\right)$. (This abbreviation shall be used throughout this paper.) Let $f_{1}(t)$ be an element of $\mathfrak{g}_{\text {out }}^{\text {orb }}$ such that $f_{1}(t) \sim X_{1} \otimes t^{-n_{1}}+O\left(t^{n}\right)$ for sufficiently large $n$. (Such $f_{1}$ exists due to the Riemann-Roch theorem. It is not difficult to construct such a function concretely. ${ }^{1}$ ) Then we may replace $X_{1}\left[-n_{1}\right]$ by $\rho_{0}\left(f_{1}(t)\right)$ :

$$
\begin{aligned}
& X_{1}\left[-n_{1}\right] \cdots X_{l}\left[-n_{l}\right]\left|\mu_{0}\right\rangle \otimes v \otimes v_{\infty} \\
= & \rho_{0}\left(f_{1}(t)\right) X_{2}\left[-n_{2}\right] \cdots X_{l}\left[-n_{l}\right]\left|\mu_{0}\right\rangle \otimes v \otimes v_{\infty} \\
\equiv & -X_{2}\left[-n_{2}\right] \cdots X_{l}\left[-n_{l}\right]\left|\mu_{0}\right\rangle \otimes \\
& \otimes\left(\sum_{i=1}^{L} \rho_{i}\left(f_{1}(t)\right) v \otimes v_{\infty}+v \otimes \rho_{\infty}\left(f_{1}(t)\right) v_{\infty}\right) \quad \bmod \mathfrak{g}_{\text {out }}^{\text {orb }} .
\end{aligned}
$$

By induction on $l$, the problem is reduced to showing

$$
\left|\mu_{0}\right\rangle \otimes v \otimes v_{\infty} \equiv 0 \quad \bmod \mathfrak{g}_{\text {out }}^{\text {orb }},
$$

which immediately follows from Lemma 5.6.

To prove the second statement of Proposition 5.3, assume that $\mu_{0}$ and $\mu_{\infty}$ are dominant integral weight. Then the irreducible quotients of $M_{\mu_{*}}^{(*)}$ are expressed as

$$
L_{\mu_{*}}^{(*)}=M_{\mu_{*}}^{(*)} / \sum_{i=0}^{N-1} U\left(\mathfrak{n}_{-}\right) f_{i}^{\left\langle\mu_{*}, \alpha_{i}^{\vee}\right\rangle+1}\left|\mu_{*}\right\rangle .
$$

See (10.4.6) of $[\mathrm{K}]$. Therefore to prove (45), it is enough to show

$$
U\left(\mathfrak{n}_{-}\right) f_{i}^{\left\langle\mu_{0}, \alpha_{i}^{\vee}\right\rangle+1}\left|\mu_{0}\right\rangle \otimes M \otimes M_{\mu_{\infty}}^{(\infty)} \equiv 0 \quad \bmod \mathfrak{g}_{\text {out }}^{\text {orb }},
$$

and a similar statement with the indices " 0 " and " $\infty$ " for $\mu_{*}, M_{\mu_{*}}^{(*)}$ etc. interchanged. They are proved as above, namely by the arguments like (48) and (49), because the weight of the singular vector $f_{i}^{\left\langle\mu_{*}, \alpha_{i}^{\vee}\right\rangle+1}\left|\mu_{*}\right\rangle$ is not a dominant integral weight.

\footnotetext{
${ }^{1} \mathrm{~A}$ useful technique: for any $\mathfrak{g}$-valued function $f(t)$ with poles in $\left\{0, Q_{1}, \ldots, Q_{L}, \infty\right\}, \quad f(t)+\operatorname{Ad} \gamma\left(f\left(\varepsilon^{-1} t\right)\right)+(\operatorname{Ad} \gamma)^{2}\left(f\left(\varepsilon^{-2} t\right)\right)+\cdots+$ $(\operatorname{Ad} \gamma)^{N-1}\left(f\left(\varepsilon^{-N+1} t\right)\right) \in \mathfrak{g}_{\text {out }}^{\text {orb }}$.
} 
Proof of Lemma 5.6. Since $\kappa$ is not a dominant integral weights, there is an index $i(0 \leqq i \leqq N-1)$ such that $\kappa\left(\alpha_{i}^{\vee}\right)$ is not a non-negative integer. By an easy calculation, we have

$$
e_{i}^{n} f_{i}^{n} v_{\kappa}=c v_{\kappa}, \quad c=n ! \prod_{l=1}^{n}\left(\kappa\left(\alpha_{i}^{\vee}\right)-l+1\right),
$$

for any $n \in \mathbb{N}$. Note that the constant $c$ never vanishes.

Let $e(t)$ be an element of $\mathfrak{g}_{\text {out }}^{\text {orb }}$ such that: (1) $e(t) \sim \phi_{0}^{-1}\left(e_{i}\right)+O\left(t^{n}\right)$ for sufficiently large $n$; $(2) \rho_{\infty}(e(t)) v_{\infty}=0$ (i.e., $e(t)$ has a zero of large order at $t=\infty)$. Such an element can be constructed in the form $X \otimes F(t)$, where $X=E_{i, i+1}(i=1, \ldots, N-1)$ or $X=E_{N, 1}(i=0)$ and $F(t)$ is a rational function. Hence we can rewrite $v_{\kappa} \otimes v \otimes v_{\infty}$ modulo $\mathfrak{g}_{\text {out }}^{\text {orb }}$ as follows (cf. p.479 of [TUY]):

$$
\begin{aligned}
& v_{\kappa} \otimes v \otimes v_{\infty} \\
& =c^{-1} \rho_{0}\left(e_{i}^{n} f_{i}^{n}\right) v_{\kappa} \otimes v \otimes v_{\infty} \\
& =c^{-1} \rho_{0}\left(e(t)^{n} f_{i}^{n}\right) v_{\kappa} \otimes v \otimes v_{\infty} \\
& \equiv(-1)^{n} c^{-1} \sum_{n_{1}+\cdots+n_{L}=n} \frac{n !}{n_{1} ! \cdots n_{L} !} \rho_{0}\left(f_{i}^{n}\right) v_{\kappa} \otimes \prod_{j=1}^{L} \rho_{j}(e(t))^{n_{j}} v \otimes v_{\infty} .
\end{aligned}
$$

Recall that $\rho_{j}(e(t))=\rho_{j}(X \otimes F(t))$ is locally nilpotent on $M_{j}$ (Corollary 1.4.6 of [TUY]). Thus the right hand side of (53) is 0 for large $n$, which completes the proof of the lemma.

Because of the difference of the sign in (43) and (44) and the fact that $\operatorname{Ad} \beta$ is a Dynkin automorphism, if $\tilde{\lambda}$ is a dominant integral weight for $\hat{\mathfrak{g}}^{(0)}, \tilde{\lambda}^{\prime}$ is a dominant integral weight for $\hat{\mathfrak{g}}^{(\infty)}$ and vice versa. (See (39).)

Corollary 5.7. Under the same assumption as Proposition 5.3 we have

$$
\mathrm{CC}_{\text {trig }}(M) \cong \bigoplus_{\lambda \in \operatorname{wt}(V), \tilde{\lambda}: \text { dom. int. }} \mathrm{CC}_{\text {orb }}\left(L_{\tilde{\lambda}}^{(0)} \otimes M \otimes L_{\tilde{\lambda}^{\prime}}^{(\infty)}\right),
$$

where "dom. int." means "dominant integral weight". Similarly the decomposition (38) becomes

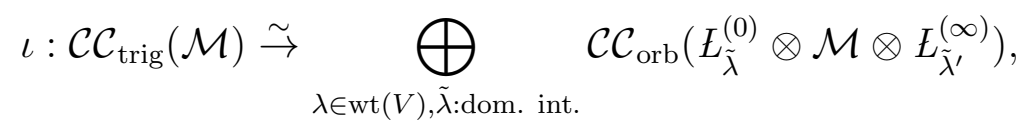

where $E_{\mu}^{(*)}=L_{\mu}^{(*)} \otimes \mathcal{O}_{S_{0}}(*=0, \infty)$. 


\section{LOCALLY FREENESS}

The main theorem of this paper is proved in this section. We show that $\mathcal{C C}$ is locally free at the discriminant locus, $S_{0}=\{q=0\} \subset S$, provided that all modules inserted are integrable highest weight modules. Thus, combining the result in $[\mathrm{KT}$, we have locally freeness of the sheaf of conformal coinvariants and consequently locally freeness of its dual, the sheaf of conformal blocks. Corresponding statement for the Weyl modules has been proved in Proposition 4.2.

The arguments in this section is parallel to those in $\S 7.3$ of [TK].

We assume the condition (35) for $\mathcal{M}$. Moreover we assume that all $M_{i}$ 's are integrable highest weight modules. In particular, the level $k$ is a non-negative integer.

Theorem 6.1. The sheaf $\mathcal{C C}(\mathcal{M})$ and hence the sheaf $\mathcal{C B}(\mathcal{M})$ are locally free $\mathcal{O}_{S}$-sheaves.

The rest of the paper is devoted to the proof of this theorem. The main strategy of the proof is the same as that of [TK]. See also [SU], [NT] and [U]:

- Since $\mathcal{C C}(\mathcal{M})$ is coherent, it is sufficient to prove that each stalk $\mathcal{C C}(\mathcal{M})_{s}(s \in S)$ is a free $\mathcal{O}_{S, s}$-module.

- We proved the locally freeness of $\mathcal{C C}(\mathcal{M})$ on the non-singular part $S \backslash S_{0}$ in [KT]. Thus we have only to prove the case $s \in S_{0}$.

- When $s \in S_{0}$, we prove that the stalk of the completion of $\mathcal{C C}(\mathcal{M})$ at $s$ is isomorphic to $\mathcal{C C}_{\text {trig }}(\mathcal{M})_{s}[[q]]$.

- $\mathcal{C C}(\mathcal{M})_{s}$ is a free $\mathcal{O}_{S, s}$-module because of Proposition 5.1 and the faithfully flatness of the completion functor.

We define completion of the sheaf $\mathcal{C C}(\mathcal{M})$ along the divisor $S_{0}$ of $S$ by taking completion of each ingredient of the definition (33). Let $\hat{\mathcal{O}}_{S / S_{0}}$ be the completion of $\mathcal{O}_{S}$ along $S_{0}$ :

$$
\hat{\mathcal{O}}_{S / S_{0}}:=\underset{n \rightarrow \infty}{\operatorname{proj} \lim } \mathcal{O}_{S} / \mathfrak{m}_{S_{0}}^{n}
$$

$\left(\mathfrak{m}_{S_{0}}\right.$ is the definining ideal of $S_{0}$.) As an $\mathcal{O}_{S_{0}}$-module, it is isomorphic to the ring of formal power series: $\hat{\mathcal{O}}_{S / S_{0}} \cong \mathcal{O}_{S_{0}}[[q]]$. The completion of $\mathcal{M}=M \otimes \mathcal{O}_{S}$ is obviously

$$
\widehat{\mathcal{M}}:=\mathcal{M} \otimes_{\mathcal{O}_{S}} \hat{\mathcal{O}}_{S / S_{0}} \cong \mathcal{M}_{S_{0}}[[q]]
$$

where $\mathcal{M}_{S_{0}}:=M \otimes \mathcal{O}_{S_{0}}$. The $\mathcal{O}_{S^{-}}$Lie algebra $\mathfrak{g}_{\mathfrak{x} \text {,out }}$ acts on $\widehat{\mathcal{M}}$ naturally as follows: a germ $f(P)$ of $\mathfrak{g}_{\mathfrak{x} \text {,out }}$ at $\left(q=0 ; Q_{1}, \ldots, Q_{L}\right) \in S_{0}$ is 
expanded at $\left(q=0 ;(0,0,0)_{k} ; Q_{1}, \ldots, Q_{L}\right) \in \tilde{\mathfrak{X}}$ in terms of the coordinates $\left(q ; x_{k}, y_{k}\right)_{k}$ as

$$
\begin{aligned}
f(P) & =\sum_{m, n=0}^{\infty} f_{k, m, n}(s) x_{k}^{m} y_{k}^{n} \\
& =\sum_{m, n=0}^{\infty} f_{k, m, n}(s) x_{k}^{m-n} q^{n}=\sum_{m, n=0}^{\infty} f_{k, m, n}(s) y_{k}^{n-m} q^{m} \\
& =\sum_{n=0}^{\infty} f_{k, n, x}\left(s, x_{k}\right) q^{n}=\sum_{m=0}^{\infty} f_{k, m, y}\left(s, y_{k}\right) q^{m},
\end{aligned}
$$

where $f_{k, m, n}(s) \in \mathfrak{g} \otimes \mathcal{O}_{S_{0}}\left(s \in S_{0}\right)$ and

$$
f_{k, n, x}\left(s, x_{k}\right)=\sum_{m=0}^{\infty} f_{k, m, n}(s) x_{k}^{m-n}, \quad f_{k, m, y}\left(s, y_{k}\right)=\sum_{n=0}^{\infty} f_{k, m, n}(s) y_{k}^{n-m} .
$$

Note that the periodicity condition (27) implies (60)

$$
\operatorname{Ad} \gamma\left(f_{k, m, n}(s)\right)=\varepsilon^{m-n} f_{k, m, n}(s), \quad \operatorname{Ad} \beta\left(f_{k, m, n}(s)\right)=f_{k+1, m, n}(s),
$$

and hence $f_{k, n, x}\left(s, x_{k}\right)$ and $f_{k, m, y}\left(s, y_{k}\right)$ are meromorphic (rational) function on $\mathbb{P}^{1}$ with quasi-periodicity

$$
\begin{aligned}
& f_{k, n, x}\left(s, \varepsilon x_{k}\right)=\operatorname{Ad} \gamma\left(f_{k, n, x}\left(s, x_{k}\right)\right), \\
& f_{k, m, y}\left(s, \varepsilon^{-1} y_{k}\right)=\operatorname{Ad} \gamma\left(f_{k, m, y}\left(s, y_{k}\right)\right), \\
& f_{k+1, n, x}\left(s, x_{k}\right)=\operatorname{Ad} \beta\left(f_{k, n, x}\left(s, x_{k}\right)\right), \\
& f_{k+1, m, y}\left(s, y_{k}\right)=\operatorname{Ad} \beta\left(f_{k, m, y}\left(s, y_{k}\right)\right),
\end{aligned}
$$

the poles of which are in the divisor

$$
* \tilde{D}_{0}:=\sum_{i=1}^{L} \sum_{j \in \mathbb{Z} / N \mathbb{Z}} *\left[\varepsilon^{j} Q_{i}\right]+*[0]+*[\infty] .
$$

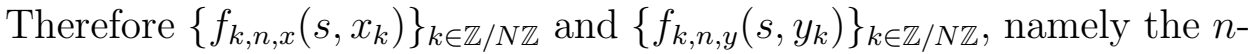
th coefficients of the expansion

$$
f(P)=\sum_{n=0}^{\infty} f_{n}(P) q^{n}
$$

define a section $f_{n}(P)$ of $\left.\mathfrak{g}_{\mathfrak{X}}^{\mathrm{tw}}\right|_{\pi_{\mathfrak{X} / S}^{-1}\left(S_{0}\right)}$ with poles at $Q_{1}, \ldots, Q_{L}, 0, \infty$. See (23) and (27). The action of $f(P)$ on $v \otimes g \in \widehat{\mathcal{M}}\left(v \in \mathcal{M}, g \in \hat{\mathcal{O}}_{S / S_{0}}\right)$ 
is defined by

$$
f(P) \cdot(v \otimes g):=\sum_{i=1}^{L} \sum_{n=0}^{\infty} \rho_{i}\left(f_{n}(P)\right) v \otimes q^{n} g .
$$

Here $\rho_{i}$ denotes the usual action of the Laurent expansion of $f_{n}(P)$ at $Q_{i}$ on $M_{i}$. The space of coinvariants of $\widehat{\mathcal{M}}$ with respect to this action is the completion of $\mathcal{C C}(\mathcal{M})$ :

$$
\widehat{\mathcal{C C}}(\mathcal{M}):=\widehat{\mathcal{M}} / \mathfrak{g}_{\mathfrak{X}, \text { out }}(\widehat{\mathcal{M}})
$$

\section{Lemma 6.2.}

$$
\widehat{\mathcal{C C}}(\mathcal{M}) \cong \mathcal{C C}(\mathcal{M}) \otimes_{\mathcal{O}_{S}} \hat{\mathcal{O}}_{S / S_{0}}
$$

Proof. By definition, we have an exact sequence

$$
\mathfrak{g}_{\mathfrak{X}, \text { out }} \otimes \mathcal{M} \rightarrow \mathcal{M} \rightarrow \mathcal{C C}(\mathcal{M}) \rightarrow 0 .
$$

Tensoring $\hat{\mathcal{O}}_{S / S_{0}}$ we obtain an exact sequence

$$
\left(\mathfrak{g}_{\mathfrak{X}, \text { out }} \otimes \mathcal{M}\right)[[q]] \rightarrow \widehat{\mathcal{M}} \rightarrow \mathcal{C C}(\mathcal{M}) \otimes \hat{\mathcal{O}}_{S / S_{0}} \rightarrow 0
$$

It is sufficient to show that the image of the map $\left(\mathfrak{g}_{\mathfrak{x} \text {,out }} \otimes \mathcal{M}\right)[[q]] \rightarrow \widehat{\mathcal{M}}$ is $\mathfrak{g}_{\mathfrak{X} \text {,out }}(\widehat{\mathcal{M}})$ defined by the action (64). This is almost trivial since $f(P) \cdot v$ for $f(P) \in \mathfrak{g}_{\mathfrak{X} \text {,out }}$ and $v \in \mathcal{M}$ is expressed as

$$
f(P) \cdot v=\sum_{i=1}^{L} \sum_{n=0}^{\infty} q^{n} \rho_{i}\left(f_{n}(P)\right) v
$$

because of the expansion (63).

Next step is to make a completion of the isomorphism (55). For this purpose we need a lemma on Verma modules of $\hat{\mathfrak{g}}^{(0)}$ and $\hat{\mathfrak{g}}^{(\infty)}$. Note that the Verma module $M_{\mu}^{(*)}$ of $\hat{\mathfrak{g}}^{(*)}(*=0, \infty)$ is graded by the degree:

$$
M_{\mu}^{(*)}=\bigoplus_{d \geq 0} M_{\mu}^{(*)}(d), \quad M_{\mu}^{(*)}(d):=\sum_{n_{1}+\cdots+n_{l}=d} \mathbb{C} X_{1}\left[-n_{1}\right] \cdots X_{l}\left[-n_{l}\right]|\mu\rangle,
$$

where $|\mu\rangle$ 's are the highest weight vectors of $M_{\mu}^{(*)}$ and $X_{i}\left[-n_{i}\right] \in \hat{\mathfrak{g}}^{(*)}$ $\left(X_{i} \in \mathfrak{g}, n_{i} \in \mathbb{N}\right)$.

Lemma 6.3. (i) For any $\mu \in \mathfrak{h}^{*}$ there exists a pairing between the Verma modules $M_{\mu \circ \mathrm{Ad} \beta}^{(0)}$ and $M_{-\mu}^{(\infty)}$ :

$$
M_{\mu \circ \mathrm{Ad} \beta}^{(0)} \times M_{-\mu}^{(\infty)}:(u, v) \mapsto\langle u, v\rangle \in \mathbb{C},
$$


which satisfies $\langle\mid \mu \circ \operatorname{Ad} \beta\rangle,|-\mu\rangle\rangle=1$ and

$$
\langle X[n] u, v\rangle+\langle u, \operatorname{Ad} \beta(X)[-n] v\rangle=0,
$$

for any $u \in M_{\mu \circ \mathrm{Ad} \beta}^{(0)}, v \in M_{-\mu}^{(\infty)}, X \in \mathfrak{g}$ and $n \in \mathbb{Z}$.

(ii) $\left\langle M_{\mu \circ \mathrm{Ad} \beta}^{(0)}(n), M_{-\mu}^{(\infty)}\left(n^{\prime}\right)\right\rangle=0$ if $n \neq n^{\prime}$.

(iii) The radical $R^{(0)}=\left\{u \in M_{\mu \circ \mathrm{Ad} \beta}^{(0)} \mid\langle u, v\rangle=0\right.$ for all $\left.v \in M_{-\mu}^{(\infty)}\right\}$ is the largest proper submodule of $M_{\mu \circ \mathrm{Ad} \beta}^{(0)}$. Similarly the radical in $M_{-\mu}^{(\infty)}$ is the largest proper submodule. Hence the pairing descends to a non-degenerate pairing between the irreducible quotients $L_{\mu \circ \mathrm{Ad} \beta}^{(0)}$ and $L_{-\mu}^{(\infty)}$.

Proof. Let $\nu$ be the anti-isomorphism

$$
\nu: U \hat{\mathfrak{g}}^{(0)} \ni X[n]=X \otimes t^{n} \mapsto-X \otimes t^{n}=-X[-n] \in U \hat{\mathfrak{g}}^{(\infty)}, \quad \nu(\hat{k})=\hat{k} .
$$

This induces a linear isomorphism $\nu_{\beta}: M_{\mu \circ \mathrm{Ad} \beta}^{(0)} \rightarrow \operatorname{Hom}_{\mathbb{C}}\left(M_{-\mu}^{(\infty)}, \mathbb{C}\right)$ defined by

$$
\nu_{\beta}(x|\mu \circ \operatorname{Ad} \beta\rangle)=\langle-\mu| \nu(\operatorname{Ad} \beta(x)),
$$

where $\langle-\mu|$ is the generating vector of the right $\hat{\mathfrak{g}}^{(\infty)}$-module $\operatorname{Hom}_{\mathbb{C}}\left(M_{-\mu}^{(\infty)}, \mathbb{C}\right)$, normalised by $\langle-\mu \mid-\mu\rangle=1$. We define the pairing $\langle$,$\rangle by$

$$
\left\langle v, v^{\prime}\right\rangle:=\nu_{\beta}(v) v^{\prime}
$$

Straightforward computation shows that for $x \in U \hat{\mathfrak{g}}^{(0)}$ we have

$$
\left\langle x v, v^{\prime}\right\rangle=\left\langle v, \nu(\operatorname{Ad} \beta(x)) v^{\prime}\right\rangle,
$$

which means (71) for $x=X[n]$.

(ii) follows from the construction.

(iii) is proved in the same way as Proposition 3.26 of [Wa].

Let $\left\{e_{\lambda, d, i}\right\}$ be a basis of $L_{\tilde{\lambda}}^{(0)}(d)$ and $\left\{e_{\lambda, d}^{i}\right\}$ be its dual basis of $L_{\tilde{\lambda}^{\prime}}^{(\infty)}(d)$ with respect to $\langle$,$\rangle .$

Proposition 6.4. There exists an isomorphism

$$
\hat{\imath}: \widehat{\mathcal{C C}}(\mathcal{M}) \rightarrow \bigoplus_{\lambda \in \operatorname{wt}(V), \tilde{\lambda}: \text { dom. int. }} \mathcal{C C}_{\text {orb }}\left(E_{\tilde{\lambda}} \otimes \mathcal{M} \otimes E_{\tilde{\lambda}^{\prime}}\right)[[q]],
$$

of $\hat{\mathcal{O}}_{S / S_{0}}$-modules defined by

$$
\hat{\iota}([v]):=\bigoplus_{\lambda}\left[\sum_{d=0}^{\infty} \sum_{i} e_{\lambda, d, i} \otimes v \otimes e_{\lambda, d}^{i}\right] q^{d},
$$


for $v \in \mathcal{M}$.

Proof. First we prove the well-definedness of (177), for which it is enough to show the well-definedness of its component $\iota_{\lambda}: \widehat{\mathcal{C C}}(\mathcal{M}) \rightarrow \mathcal{C C}_{\text {orb }}\left(\mathcal{M}_{\tilde{\lambda}} \otimes\right.$ $\left.\mathcal{M} \otimes \mathcal{M}_{\tilde{\lambda}^{\prime}}\right)[[q]]$, namely,

$$
\sum_{d=0}^{\infty} \sum_{i} e_{\lambda, d, i} \otimes f(P) \cdot v \otimes e_{\lambda, d}^{i} q^{d} \in \mathfrak{g}_{\mathrm{out}}^{\mathrm{orb}}\left(\mathcal{M}_{\tilde{\lambda}} \otimes \mathcal{M} \otimes \mathcal{M}_{\tilde{\lambda}^{\prime}}\right)
$$

for $f(P) \in \mathfrak{g}_{\mathfrak{X} \text {,out }}$ and $v \in \mathcal{M}$. Since

$$
f(P) \cdot\left(\sum_{d=0}^{\infty} \sum_{i} e_{\lambda, d, i} \otimes v \otimes e_{\lambda, d}^{i}\right) q^{d} \in \mathfrak{g}_{\mathrm{out}}^{\mathrm{orb}}\left(\mathcal{M}_{\tilde{\lambda}} \otimes \mathcal{M} \otimes \mathcal{M}_{\tilde{\lambda}^{\prime}}\right),
$$

we have only to show that the left hand side of (178) is equal to the left hand side of (79), which is equivalent to an equation in $L_{\tilde{\lambda}} \otimes L_{\tilde{\lambda}^{\prime}}$ :

$$
\sum_{d=0}^{\infty} \sum_{i}\left(\rho_{0}(f(P)) \cdot e_{\lambda, d, i} \otimes e_{\lambda, d}^{i}+e_{\lambda, d, i} \otimes \rho_{\infty}(f(P)) \cdot e_{\lambda, d}^{i}\right) q^{d}=0
$$

Recall that the germ of $f(P)$ at 0 and the germ at $\infty$ is related by $f(P)_{\infty}=\operatorname{Ad} \beta\left(f(P)_{0}\right)$. See (27) of this paper or (18) of [T]. Hence using the expansion (58) and the invariance (71) of the pairing, we can show (80) in the same way as the proof of Claim 3 in the proof of Theorem 6.2.1 in TUY]. Thus $\hat{\imath}$ is well-defined.

Obviously the $q=0$ part of $\hat{\iota}$ is the isomorphism $\iota$, (55). Therefore by termwise approximation (in analytic language) or, in other words, by Nakayama's lemma (in algebraic language), $\hat{\imath}$ is shown to be an isomorphism.

With these preparations, the proof of the locally freeness of $\mathcal{C C}(\mathcal{M})$ goes as follows. As is mentioned after the statement of Theorem 6.1. it is enough to prove that the stalk $\mathcal{C C}(\mathcal{M})_{s}$ at $s \in S_{0}$ is a free $\mathcal{O}_{S, s}$-module. Since $\widehat{\mathcal{C C}}(\mathcal{M})_{s}$ is isomorphic to $\mathcal{C C}(\mathcal{M})_{s} \otimes_{\mathcal{O}_{S, s}} \otimes \hat{\mathcal{O}}_{S / S_{0}, s}$ (Lemma 6.2) and to $\bigoplus_{\lambda} \mathcal{C C}_{\text {orb }}\left(\mathrm{E}_{\tilde{\lambda}} \otimes \mathcal{M} \otimes \mathrm{E}_{\tilde{\lambda}^{\prime}}\right)_{s}[[q]]$ (Proposition 6.4), we have an isomorphism

$$
\mathcal{C C}(\mathcal{M})_{s} \otimes_{\mathcal{O}_{S, s}} \hat{\mathcal{O}}_{S / S_{0}, s} \cong \bigoplus_{\lambda} \mathcal{C} \mathcal{C}_{\text {orb }}\left(\mathrm{E}_{\tilde{\lambda}} \otimes \mathcal{M} \otimes \mathrm{E}_{\tilde{\lambda}^{\prime}}\right)_{s}[[q]]
$$

The right hand side of (81) being a free $\hat{\mathcal{O}}_{S / S_{0}, s}$-module (Proposition 5.1 (ii) and Proposition 5.3), faithfully flatness of $\hat{\mathcal{O}}_{S / S_{0}, s}$ over $\mathcal{O}_{S, s}$ implies that $\mathcal{C C}(\mathcal{M})_{s}$ is a free $\mathcal{O}_{S, s}$-module. Thus Theorem 6.1 is proved. 


\section{Concluding COMments}

We have proved locally freeness of $\mathcal{C C}(\mathcal{M})$ in two cases; Weyl module case (Proposition 4.2) and integrable highest weight module case (Theorem 6.1). A few comments are in order:

- In the Weyl module case, $\mathcal{C C}(\mathcal{M}) \cong V \otimes \mathcal{O}_{S}$ as shown in the proof of Proposition 4.2 and the rank of $\mathcal{C C}(\mathcal{M})$ is $\operatorname{dim} V$.

- In the integrable highest module case, the rank is computed by further degenerating the orbifold. Degeneration of the type $Q_{i} \rightarrow Q_{j}$ should be considered in the same way as in TUY] or [NT. The final results of the degeneration is a combination of the three-punctured orbifold $\mathbb{P}^{1} / C_{N}$. In principle a Verlindetype formula would be obtained in this way.

- In $\mathrm{KT}$ we have shown that $\mathcal{C C}(\mathcal{M})$ has a flat connection. It has a regular singularity along $S_{0}=\{q=0\}$, which is easily deduced from the explicit form of the connection, Theorem 5.9 of $[\mathrm{KT}$. Hence there is a one-to-one correspondence between flat sections around $S_{0}$ and its restriction to $S_{0}$ or, in other words, the "initial value" at $S_{0}$ because of the locally flatness.

\section{ACKNOWLEDGMENTS}

The author expresses his gratitude to Akihiro Tsuchiya who explained details of [TUY] and [NT], Toshiro Kuwabara who showed the manuscript of TK (the best guide to TUY for $\mathbb{P}^{1}$ case) before publishing, Michio Jimbo, Gen Kuroki, Tetsuji Miwa, Hiroyuki Ochiai, Kiyoshi Ohba, Nobuyoshi Takahashi, Tomohide Terasoma and Yasuhiko Yamada for discussion and comments.

The atmosphere and environment of Institute for Theoretical and Experimental Physics (Moscow, Russia) and the conference "Infinite Dimensional Algebras and Integrable Systems" (Faro, Portugal) were very important. The author thanks their hospitality.

\section{REFERENCES}

[BD] A. A. Belavin, V. G. Drinfeld, Solutions of the classical Yang-Baxter equations for simple Lie algebras. Funkts. Anal. i ego Prilozh. 16-3, 1-29 (1982) (in Russian); Funct. Anal. Appl. 16, 159-180 (1982) (English transl.)

[E] P. I. Etingof, Representations of affine Lie algebras, elliptic $r$-matrix systems, and special functions. Comm. Math. Phys. 159, 471-502 (1994).

[FFR] B. Feigin, E. Frenkel, N. Reshetikhin, Gaudin model, Bethe Ansatz and critical level. Commun. Math. Phys. 166, 27-62 (1994)

[K] V. G. Kac, Infinite dimensional Lie algebras, 3rd Edition, Cambridge University Press 1990. 
[KL] D. Kazhdan, G. Lusztig, Tensor structures arising from affine Lie algebras. I, II, J. Amer. Math. Soc. 6, 905-948, 949-1011 (1993).

[KT] G. Kuroki, T. Takebe, Twisted Wess-Zumino-Witten models on elliptic curves. Comm. Math. Phys. 190, 1-56 (1997).

[NT] K. Nagatomo, A. Tsuchiya, Conformal field theories associated to regular chiral vertex operator algebras I: theories over the projective line, math.QA/0206223

[SU] Y. Shimizu, K. Ueno, Moduli theory III, (Iwanami, Tokyo, 1999) Gendai Suugaku no Tenkai series (in Japanese); Advances in moduli theory, Translations of Mathematical Monographs, 206. Iwanami Series in Modern Mathematics, American Mathematical Society, Providence, U.S.A. (2002) (English translation)

[T] T. Takebe, Trigonometric Degeneration and Orbifold Wess-Zumino-Witten Model. I In the Proceedings of the 6th International workshop on Coformal and Integrable models, Chernogolovka, Sep. 2002, International Journal of Modern Physics, A, 19, Supplement, 418-435 (2004)

[TK] A. Tsuchiya, T. Kuwabara, Introduction to Conformal Field Theory, to appear as MSJ Suugaku Memoir of Mathematical Society of Japan.

[TUY] A. Tsuchiya, K. Ueno, Y. Yamada, Conformal field theory on universal family of stable curves with gauge symmetries. In Integrable systems in quantum field theory and statistical mechanics, Adv. Stud. Pure Math. 19, 459-566 (1989).

[U] K. Ueno, On conformal field theory, In Vector bundles in algebraic geometry (Durham, 1993), ed. by N. J. Hitchin, P. E. Newstead and W. M. Oxbury, London Math. Soc. Lecture Note Ser. 208, (Cambridge Univ. Press, Cambridge, 1995) pp. 283-345,

[Wa] M. Wakimoto, Infinite-dimensional Lie algebras, (Iwanami, Tokyo, 1999) Gendai Suugaku no Tenkai series (in Japanese); Translations of Mathematical Monographs, 195, Iwanami Series in Modern Mathematics, American Mathematical Society, Providence, U.S.A. (2001) (English translation by K. Iohara)

[Wo] S. Wolpert, On the homology of the moduli space of stable curves. Ann. of Math. 118, 491-523 (1983).

[Z] A. B. Zamolodchikov, Exact solutions of conformal field theory in two dimensions and critical phenomena. Rev. Math. Phys. 1 197-234 (1989). (Translated from the Russian by Y. Kanie.)

Department of Mathematics, Ochanomizu University, Otsuka 2-1-1, BUNKYO-KU, TOKYO, 112-8610, JAPAN

E-mail address: takebe@math.ocha.ac.jp 